\title{
Géolinguistique
}

15 | 2015

La géographie linguistique au Brésil

\section{L'Atlas des parlers de Bahia (APFB) et la géographie linguistique au Brésil}

The Atlas of Bahian's Dialects (APFB) and Linguistic Geography in Brazil

Carlota Ferreira et Dinah Callou

\section{(2) OpenEdition}

Journals

Édition électronique

URL : http://journals.openedition.org/geolinguistique/595

DOI : 10.4000/geolinguistique.595

ISSN : 2650-8176

Éditeur

UGA Éditions/Université Grenoble Alpes

Édition imprimée

Date de publication : 1 décembre 2015

Pagination : 25-37

ISBN : 978-2-84310-317-9

ISSN : 0761-9081

Référence électronique

Carlota Ferreira et Dinah Callou, "L'Atlas des parlers de Bahia (APFB) et la géographie linguistique au Brésil », Géolinguistique [En ligne], 15 | 2015, mis en ligne le 15 février 2019, consulté le 05 novembre 2020. URL : http://journals.openedition.org/geolinguistique/595 ; DOI : https://doi.org/10.4000/ geolinguistique. 595 


\title{
L'Atlas des parlers de Bahia (APFB) et la géographie linguistique au Brésil
}

\author{
Carlota Ferreira \\ Universidade Federal da Bahia (Brésil) \\ Dinah Callou \\ Universidade Federal do Rio de Janeiro - CNPq (Brésil)
}

\section{Résumé}

Cet article se veut être une réflexion sur l'importance du premier atlas régional brésilien (AFPB) pour le développement de la géographie linguistique au Brésil. On discute (i) les raisons pour lesquelles ce n'est que dans les années 1960 que les recherches dialectales deviennent une réalité; (ii) la méthode utilisée pour le recueil des échantillons représentatifs des parlers régionaux; et (iii) l'incorporation, tout au long du temps, d'autres variables sociales - au-delà de la différenciation diatopique - dans la présentation des résultats.

\section{Mots-clés}

Atlas linguistique brésilien, parlers régionaux, variation diatopique, variation social.

\section{Abstract}

This paper brings some reflections on the role of the publication of the first regional Brazilian Linguistic Atlas (APFB) to the development of dialectal studies in our country. It discusses (i) the reasons why dialectal researches become reality only at the beginning of the 60's; (ii) the scientific methodology of gathering representative oral speech samples; and 
(iii) the incorporation across time of other social variables-besides diatopic differentiation-on displaying the results.

\section{Keywords}

Brazilian Linguistic Atlas, regional dialects, diatopic variation, social variation.

Du premier atlas régional brésilien (APFB, 1963) à l'Atlas linguistique du Brésil (ALiB, 2014) cinquante ans ont passé. Comme il fallait s'y attendre, beaucoup de choses se sont modifiées dans ce laps de temps, non seulement la conception même d'un atlas - de nos jours multidimensionnel - mais aussi son exécution pratique. Néanmoins, si, d'un côté, le premier présente des failles, inévitables à l'époque, surtout l'absence d'enregistrement des enquêtes sur magnétophone - la transcription phonétique des réponses au questionnaire était faite in loco -, de l'autre, il reste une avancée dans le domaine de la méthodologie de préparation des enquêteurs, qui n'a toujours pas été remplacée à l'heure actuelle.

Comme chacun le sait, un atlas linguistique est fondamentalement un ensemble de matériaux linguistiques, un grand dictionnaire dialectal qui vise à présenter, de manière scientifique et vivante, sur chaque carte, les différentes variantes d'un mot ou d'une petite phrase sur un territoire plus ou moins étendu. Comme les mots et les phrases objets de la recherche sont choisis à l'avance, suite à une étude minutieuse des formes culturelles d'une communauté dont la langue est l'expression, l'inventaire, même incomplet, permet une ample vision des traits essentiels du lexique : sa répartition géographique, sa vitalité, l'histoire des termes, les voyages des mots, l'évolution des formes grammaticales, les différences de prononciation, les relations des dialectes et des parlers avec la langue cultivée. Et comme le mot est étroitement lié à la chose qu'il désigne, l'atlas permet aussi de vérifier le comportement des formes et des réalités culturelles.

Dans un pays de cette étendue, il serait impossible d'envisager d'emblée d'élaborer un atlas national, il est préférable de programmer une série d'atlas régionaux, pour ensuite penser à l'œuvre gigantesque qui recouvre la totalité du territoire brésilien. Tel est le choix qui fut fait.

L'un des objectifs de ce texte est de provoquer quelques réflexions sur le rôle de la publication du premier atlas régional brésilien (APFB), sur le développement de la géographie linguistique au Brésil. On discute les raisons pour lesquelles ce n'est que dans les années 1960 que les recherches dialectales sont devenues réalité, utilisant une méthodologie scientifique 
de relevés représentatifs du parler, et le fait que d'autres variables sociales que la diatopique y aient été incorporées avec le temps.

L'Atlas des parlers de Bahia constitue un point de repère dans l'histoire des études dialectales. On peut assurément affirmer que ce qu'on nomme l'APFB, le premier atlas linguistique régional brésilien, imaginé et réalisé sous la coordination de Nelson Rossi, marque le début des études géolinguistiques et va même au-delà, en introduisant la recherche linguistique proprement dite au Brésil, dans ce cas à l'Université fédérale de Bahia (UFBA).

Faisons une brève rétrospective. C'est à partir de la dernière décennie du $\mathrm{XIX}^{\mathrm{e}}$ siècle que la dialectologie a pris sa place d'honneur dans la linguistique, en particulier française. On attribue, en général, une importance décisive, pour le développement de la discipline, au dramatique appel de Gaston Paris, dans sa célèbre conférence Les parlers de France (1888), à recueillir les patois, en voie d'extinction par la force unificatrice du progrès culturel, car ils contenaient une partie pertinente de l'histoire du pays. L'appel fut suivi d'effet, car à partir de là surgirent plusieurs atlas commandés par l'Atlas linguistique de la France (1902-1910).

Au Brésil, jusqu'aux années 1960, les études sur la langue portugaise souffraient d'un grand retard et ceci en raison de nombreux facteurs, parmi lesquels la création tardive des Facultés de lettres. Rêvée durant des années, l'entreprise d'un atlas linguistique du Brésil fut sans cesse reportée, à cause de l'étendue territoriale du pays, de l'asymétrie de la densité démographique et du manque de ressources humaines et financières, entre autres. Le manque de moyens pour les recherches de nature linguistique et le long culte du purisme ont eu pour conséquence naturelle un désintérêt à recueillir les parlers locaux. La dialectologie brésilienne est née, en réalité, du Dialeto Caipira [Dialecte paysan], d'Amadeu Amaral, publié en 1920. Avant lui, seuls quelques lexiques régionaux existaient, le premier datant de 1852, d'Antônio Alvares Pereira Coruja.

Le travail d'Amadeu Amaral eut le mérite d'attirer l'attention sur l'importance et l'urgence d'une étude systématique de nos parlers, condamnés à périr via un nivellement progressif. Ce fut lui qui encouragea, comme il l'a reconnu, les recherches d'Antenor Nascentes sur le parler carioca (1922) et d'autres qui suivirent. En outre, sans être systématiques, les études sur les parlers brésiliens n'ont eu d'incidence, pendant longtemps, que sur de rares zones du territoire national.

Le parcours a été long pour parvenir à la préparation du premier atlas régional. Sa contribution coïncide avec l'arrivée de son auteur, en 1955, à l'Université fédérale de Bahia, venu implanter parmi nous la mentalité dialectologique nécessaire. Ce ne serait pas un atlas exhaustif, mais il fournirait 
plutôt une vision des possibles zones linguistiques, déduites pour la première fois à partir de faits de la langue recueillis systématiquement. Le titre Atlas préliminaire ne s'oppose pas à celui d'atlas définitif, c'est plutôt une espèce de bande-annonce de la réalité linguistique brésilienne. Selon les mots de son auteur, il constitue «par la nature même du tissu emmêlé qu'on essaie de débrouiller [...] un pur instantané du résultat de quatre siècles d'indiscipline générale et effrénée ${ }^{1} \gg$ (Rossi, 1965, p. 50).

\section{Historique et structure de l'étude}

L'Atlas Prévio dos Falares Baianos (Atlas préliminaire des parlers de Bahia - APFB) a été élaboré dans les années 1960-1962 avec l'aide de plusieurs collaborateurs ${ }^{2}$. Publié en 1963 par l'Institut national du livre/ MEC, à Rio de Janeiro, il fut entièrement financé par l'Université fédérale de Bahia. L'idée fut semée par Nelson Rossi qui commença un travail dialectologique au Brésil, dès son arrivée à l'UFBA, à la tête de la discipline de langue portugaise, à l'époque à la Faculté de philosophie. La rénovation de l'enseignement proposée par le professeur Nelson Rossi avait pour but d'initier les étudiants aux problèmes concrets des enquêtes linguistiques, remplaçant les cours traditionnels par des recherches sur le terrain - sans toutefois négliger la formation théorique indispensable et par conséquent d'éveiller des vocations. Entre 1956 et 1959, il instaura la pratique des sondages préliminaires à l'intérieur de Bahia, toujours en collaboration avec les étudiants. À cette époque, on réalisa quelques études basées sur des faits déjà recueillis, parmi lesquelles l'ouvrage qui se réfère aux Aspectos do léxico regional da Bahia (Aspects du lexique

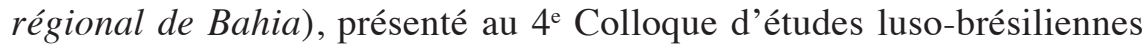
qui eut lieu à Salvador en 1959. À ce travail collaborèrent des étudiants qui allaient ensuite participer à l'APFB et il représenta le dernier coup de pouce et l'élan nécessaire pour commencer l'atlas, l'année suivante. On ne peut éviter de regarder en arrière pour voir quand et comment tout a commencé et s'est passé, pourtant il est impossible de donner une idée exacte de ce que fut cette expérience extraordinaire dans la vie d'un chercheur. Il

1. Texte original : «pela própria natureza do tecido emaranhado que se procura deslindar [...] mero instantâneo do resultado de quatro séculos de indisciplina geral desenfreada».

2. Les auteures de cet article ont fait partie de l'équipe qui a développé ce travail pionnier, de la première à la dernière étape (collaboratrices principales). Elles appartiennent à cette génération privilégiée qui était à la bonne heure, au bon endroit. 
est, cependant, important de rappeler les mots de Cintra (1983, p. 15) sur l'intérêt humain - qui transcende l'intérêt strictement scientifique - de l'étude dialectologique et «l'influence profonde et décisive qu'elle peut assumer dans l'attitude et l'orientation générale d'un linguiste qui s'y est une fois consacré ${ }^{3} »$.

Toute œuvre porte en soi le mérite d'être pionnière, mais contient de nombreuses limitations. Si nous regardons le passé, puis le présent et si nous nous projetons dans l'avenir, il est possible d'observer la distance qui existe entre ce qui a été fait, ce qui se fait et ce qui se fera. Et dans notre cas, la distance est fixée non seulement par le progrès des sciences du langage, avec ses intérêts divers et ses méthodologies précises, mais aussi par les nouvelles technologies qui servent à exploiter et à analyser les données. Ce qui reste néanmoins immuable, c'est le goût et le sérieux avec lesquels ce travail est fait. Et la publication de l'Atlas Lingüístico do Brasil (Atlas linguistique du Brésil-ALiB) en est la preuve.

\subsection{Sélection des localités et élaboration du questionnaire}

L'APFB est composé de 50 points d'enquêtes: 30 municipalités, 15 villages et 5 hameaux. Le point de départ pour le choix des localités fut la proposition d'Antenor Nascentes dans Bases para elaboração dos atlas linguísticos do Brasil (1958, p. 20). Sa suggestion se bornait à 39 points pour Bahia et ne répondait pas toujours aux présupposés théoriques du choix d'une localité (ancienneté, vie propre, isolement relatif, population numériquement représentative, en particulier) qui ont été adoptés, chaque fois que cela était possible, pour faire l'APFB, joints au critère d'équidistance $v s$ densité démographique. En 1960, l'État de Bahia était divisé en trois zones physiographiques et elles ont toutes été étudiées. Des 50 lieux finalement sélectionnés, à peine 13 coïncident avec ceux suggérés par Nascentes. Pour le choix définitif des localités de l'État de Bahia, on a également procédé à une ample lecture des œuvres des premiers voyageurs, historiens et géographes, ainsi que des ouvrages qui fournissaient des données historiques, géographiques et socio-économiques plus récentes, pour 1960. À partir des lectures, on a recensé 1076 toponymes qui furent réduits à 599 localités, fichées avec les renseignements suivants : noms actuels, noms antérieurs, référence chronologique la plus ancienne, synthèse historique, degré d'isolement, etc. Le nombre de ces toponymes fut ensuite réduit à 48 , puis passa à 50 en raison de diverses circonstances pendant la phase de réalisation des

3. Texte original : "a profunda e decisiva influência que ele pode assumir na atitude e na orientação geral de um linguista que alguma vez a ele se dedicou». 
enquêtes. Si l'on considère la superficie administrative de l'État de Bahia, force nous est de reconnaître la faible densité du réseau.

Sans modèle précédent au Brésil qui puisse servir de guide, le questionnaire utilisé par l'APFB fut élaboré à partir d'expériences dialectales antérieures, faites par divers groupes d'étudiants, et ensuite adapté à la réalité bahianaise. Dans les années 1958 et 1959, on a utilisé des questionnaires de plus de 3000 questions, divisés en quatre grands champs sémantiques - Terre, Végétaux, Homme, Animaux - soumis à des informateurs ruraux de quatre localités à l'intérieur de l'État: Bom Despacho (1958), São José das Itapororocas (après Maria Quitéria), Tanquinho et São Vicente (1959). À partir de l'analyse des réponses fournies par les informateurs de ces quatre localités, après une étude détaillée de plusieurs lexies, on élabora l'Extrait de questionnaire (EQ). Cet EQ est composé de 164 questions (élicitation indirecte), certaines subdivisées et indexées par les lettres A, B, C et D, parvenant à un total de 182 questions, regroupées selon leur proximité sémantique. C'est un questionnaire de base lexicosémantique qui permet de noter des données phonétiques à partir des diverses réalisations des lemmes.

Il est toujours bon de rappeler que les conclusions qu'on peut tirer du matériel réuni dans le volume de cartes seraient probablement et naturellement différentes si les questions et les lieux de l'enquête étaient autres. Ce que l'on peut affirmer, c'est que si le questionnaire avait été élargi, étant donné le contexte de l'époque (n'oublions pas que tout cela s'est fait il y a plus de 50 ans!), l'APFB n'aurait certainement pas été possible, du moins à ce moment-là. On peut néanmoins observer que ce sont les informateurs eux-mêmes, ceux des sondages préliminaires, qui ont, d'une certaine façon, suggéré le questionnaire.

\subsection{Préparation des enquêteurs}

La méthodologie de préparation des enquêteurs - outre Nelson Rossi, la plupart des licenciés - constitue un chapitre à part. Les licenciés qui suivirent des cours réguliers de dialectologie en dernière année de lettres (1959) furent également co-auteurs de l'ouvrage, déjà cité, Aspectos do léxico regional da Bahia et expérimentèrent le travail de terrain dans trois localités proches de Salvador. Ils participèrent, en outre, à toutes les étapes préparatoires, à savoir, le choix des localités et la préparation de l'EQ.

Le plus remarquable, fut l'intense stage d'entraînement de transcription phonétique, commencé par des exercices de transcription de mots isolés et de courts passages de langues inconnues des collaborateurs/licenciés, comme le russe et le yoruba, en passant ensuite au portugais du Portugal (standard et rural) et juste dans la phase finale au portugais du Brésil (éga- 
lement standard et rural). Il apparaît clairement qu'on a cherché à approcher progressivement la réalité qu'on pensait trouver. Partir strictement de l'inconnu éviterait toute association phonique et mentale avec des segments linguistiques connus et éviterait aussi l'interférence des habitudes orthographiques. On a juste voulu admettre deux entités présentes : les sons entendus et les signes dont on disposait pour les représenter.

Le système de transcription utilisé fut celui de Lacerda-Hammarsrtröm (1953), avec quelques adaptations au portugais du Brésil, basées sur l'IPA. On donna une emphase spéciale à la possibilité d'entendre, dans les recherches sur le terrain, les segments phoniques - des réalisations de phonèmes ou de combinaisons de phonèmes - non prévus dans le système de transcription. Dans ces cas, les enquêteurs devaient chercher des solutions pour résoudre les difficultés en décrivant verbalement ce qu'ils entendaient. Nelson Rossi se réfère aux enquêteurs dans le volume Introduction : «Il nous a paru légitime d'admettre que nous étions alors les moins apprentis possibles à tenter de faire un atlas ${ }^{4} . »(1965$, p. 20$)$

\subsection{Les informateurs et les enquêtes linguistiques}

La première enquête linguistique fut réalisée le 31 octobre 1960 et la dernière le 7 avril 1961, alors que la phase d'élaboration des cartes avait déjà débutée. La première localité, Vila de Abrantes ( $\mathrm{n}^{\circ}$ 5), fonctionna comme test en présence de tous les enquêteurs, regroupés en paires. Dans l'enquête, six informateurs furent entendus, alors que dans les enquêtes suivantes (dans 41 lieux) on n'en utilisa que deux. On interrogea un total de 100 informateurs, auxquels s'ajoutèrent 4 participants éventuels dont les réponses furent également cartographiées et identifiées.

Concernant le choix des informateurs, on a essayé d'en avoir toujours deux, de sexe et d'âge différents - entre 25 et 60 ans -, outre les exigences habituelles : être natifs de la même localité que les parents, avoir peu voyagé, analphabètes ou semi-analphabètes, critères pas toujours possibles à respecter. Les recommandations sur la manière de formuler les questions furent celles habituellement utilisées : interrogation indirecte, transcription immédiate et sans retouche ultérieure à la réponse, dose attentive d'insistance pour ne pas provoquer de fatigue ou d'abandon. On a aussi utilisé une méthodologie inhabituelle pour les travaux de cette nature. Après l'utilisation intégrale du questionnaire, certaines réponses n'ayant pas été obtenues, mais relevées dans d'autres zones, on interrogeait l'informateur

4. Texte original : «Julgamos lícito admitir que éramos então os menos aprendizes que se podia ser antes de haver tentado um atlas.» 
sur l'expression en question. La réponse donnée par le test d'identification directe a parfois été cartographiée, en fonction de la caractérisation fournie par les informateurs.

\section{4. Élaboration des cartes}

Ce thème mérite d'être mis en relief, puisqu'à cette époque-là la tâche de disposer les réponses sur la carte constituait un travail monastique : classer les transcriptions, couper, coller et répartir les réponses sur la carte à l'aide d'une pince. On ne pouvait compter, à cette époque, sur aucun moyen numérique. L'APFB se compose de 154 cartes linguistiques, presque toutes en transcription phonétique et quelques-unes en signes graphiques conventionnels, plus 44 cartes-résumés qui synthétisent les informations.

La phase d'analyse des données a montré que faire une carte n'est pas un simple travail de transcription des réponses fournies sur un cartogramme. Il faut d'abord sélectionner les réponses cohérentes avec les questions, sans ambiguïté, car les malentendus entre informateur et enquêteur ne sont pas rares, surtout en fonction de la question, de concepts plus abstraits, d'actions verbales, etc. Finalement, on renonça à obtenir $100 \%$ de garantie et à profiter du maximum. On préféra, ainsi, courir le risque d'une interprétation préliminaire des matériaux, en présentant sur les cartes des informations sélectionnées de façon critique, et donner, de préférence, au lecteur des éléments de contrôle, au moyen de notes apposées aux cartes, ce qui constituait une innovation. Les notes qui accompagnent chaque carte contiennent soit le questionnement des enquêteurs soit les réponses des informateurs, ces dernières sont sans aucun doute les plus importantes car elles élargissent les données linguistiques, non seulement au niveau du lexique et de la phonétique, mais encore au niveau de la morphosyntaxe, et transmettent mieux le milieu culturel dans lequel vit l'informateur, sa vision des choses et même sa propre langue.

Les cartes sont précédées de 30 pages qui racontent l'histoire des localités, leur situation administrative entre 1950 et 1960, suivant l'Institut brésilien de géographie et statistiques (IBGE), leur localisation spatiale dans l'État de Bahia, leur population, ainsi que des données sur les informateurs selon un schéma fixe de présentation.

\section{Une vision générale de l'APFB}

Selon Ferreira (1998), à partir d'une vision diatopique des cartes linguistiques de l'Atlas des parlers de Bahia, se dessinent des isoglosses lexicales de moindre amplitude qui indiquent, si on les confronte entre elles, l'existence de cinq sous-zones ainsi réparties : 
a) Partie de la zone de Barreiras, Baixo Médio São Francisco et Sertão de São Francisco :

- canastra pour galipette (APFB 109/109R)

- rodela pour rotule du genou (APFB 62/62R)

- nambu/lambu pour sans queue [oiseau] (APFB 113/113R)

b) Zone du Nord-Est, allant jusqu'au Sud, avec une certaine pénétration dans le Centre :

- lambedor pour sirop fait-maison (APFB 96)

- cacumbu pour outil très usagé (APFB 154)

- ovo de peru pour tâche de rousseur (APFB 75)

c) Zone du Nord-Est, Littoral Nord et Recôncavo :

- pataqueiro pour travailleur agricole utilisant la houe (APFB 23/23R)

- cobé pour sorcier (APFB 99/99R)

- mazá pour sangsue (APFB 128/128R)

d) Région Sud :

- mandraqueiro pour sorcier (APFB 99/99R)

- batoeira pour épis de maïs (APFB 28/28R)

- noruega pour brouillard (APFB 12/12R)

e) Zone du Médio São Francisco, Serra Geral et Chapada Diamantina :

- coxé pour personne qui a une jambe plus courte que l'autre (APFB 79/79R)

- pataca pour rotule du genou (APFB 62/62R)

- quém-quém pour pintade (APFB 114)

D'un autre côté, en atteignant un degré maximum de diversité lexicale, on enregistre plusieurs formes d'expression pour un même contenu, sans avoir observé une quelconque répartition par zone, comme dans le cas de la carte 32 : partie terminale de l'inflorescence du bananier.

Du point de vue phonétique, on peut déduire que /t/ et /d/ devant /i/ peuvent se palataliser à un degré plus ou moins grand dans une zone et conserver dans l'autre une articulation alvéolaire. La carte 81 en est une bonne représentation. Au sud de Bahia - en particulier sur le littoral les consonnes sont des affriquées palatales, ce qui semble caractéristique de cette zone (cartes 70, 71, 99).

À observer encore, entre autres, le fait que la forme archaïque nasalisée lu a luã (aussi luma), conservée dans d'autres régions du nord-est du pays, a été relevée dans des lieux relativement proches. La forme archaïque 
sarolha (et ses variantes), à peine enregistrée auparavant dans le Livro da Montaria [Livre d'équitation] de D. João I (Silva Neto, 1952, p. 420), s'est retrouvée dans presque tout l'État, dans le sens de humide (terre), à l'exception des zones physiographiques dénommées Zona do Cacau [Zone du Cacao] et Extremo Sul [Extrême Sud], et dans quelques lieux proches de l'État de Minas Gerais (cartes 22 et 22R).

$\mathrm{Au}$ sujet du nombre de réponses différentes à une même question, les cartes 23, 28, 35, 52, 53, 62, 63, 109 et autres méritent consultation. Ces réponses à plusieurs questions ne présentent pas toujours une répartition spatiale identique, ce qui empêche de définir des sous-zones linguistiques fixes à l'intérieur de l'État de Bahia, bien qu'il y ait parfois correspondance entre les limites et les zones [cf. cartes $35 \mathrm{R}$ et $62 \mathrm{R}$, par exemple, où l'on peut percevoir que la zone de la forme bolinete (35R) coïncide partiellement avec celle de rodela $(62 \mathrm{R})$ et celle de rodela $(35 \mathrm{R})$ avec celle de bolacha (62R)].

En somme, en ce qui concerne les aspects phonétiques et lexicaux, contenus dans un atlas, il n'est pas toujours viable d'établir un faisceau d'isoglosses déterminant des zones dialectales claires, mais on ne peut pas nier que les indices sont forts.

\section{Remarques finales}

Les atlas linguistiques révélaient, au début, un plus grand souci de la variation diatopique, mais avec le temps ils ont ajouté une dimension verticale - sociale - à la géographie - horizontale - et ont pris en compte toute variation de nature socio-culturelle, sachant qu'il y a des éléments de différenciation, même dans le dialecte rural le plus isolé.

On a longtemps discuté de la pertinence de la géographie linguistique et de l'adéquation de la méthode cartographique de Gillérion - même perfectionnée par des techniques modernes et enrichie d'apports ethnographiques - pour observer la réalité linguistique de la Romania américaine où l'on n'a jamais parlé latin. On ne pourrait obtenir de réponse à cette question avant de se demander : quelles sont les finalités d'une recherche dialectologique dans les pays américains, ou plutôt, pour limiter la question au cadre national, quelles informations linguistiques désirions-nous obtenir de ces enquêtes sur le territoire brésilien?

Il n'y a aucun doute que la variété verticale du langage a assumé de nouveaux aspects sur le continent américain où la croissance vertigineuse de certaines villes a provoqué un profond changement sur l'échiquier linguistique régional, rapidement nivelé par l'influence colossale de la norme qui en émane. Bien qu'on reconnaisse la pertinence de la dialectologie ver- 
ticale, de la sociolinguistique, on ne doit pas sous-estimer la contribution apportée par la dialectologie spatiale à la connaissance de la réalité linguistique des pays américains. La différenciation régionale, encore très sensible dans certains lieux, ne pouvait être montrée de façon satisfaisante que dans un atlas linguistique, seul moyen de voir clairement les dénivelés temporels de l'usage de la langue - les zones d'archaïsmes et de néologismes. Les dialectologies horizontale et verticale ne sont donc pas mutuellement exclusives. Au contraire, elles se complètent dans la description de l'état actuel d'une langue et, selon nous, peuvent aussi servir à l'étude diachronique des variétés linguistiques de petite histoire, ainsi que nous le montre une analyse des conditions dans lesquelles la langue portugaise s'est développée en Amérique. La variation existante de nos jours dans le portugais du Brésil, qui nous permet de reconnaître une pluralité de parlers, est le fruit de la dynamique populationnelle et de la nature du contact des divers groupes ethniques et sociaux aux différentes périodes de notre histoire.

On dit que l'un des objectifs d'un atlas linguistique est d'offrir - dans ce cas particulier - un portrait du Brésil, c'est-à-dire rendre compte de la diversité existante, ou plutôt de la dialectalisation du portugais, afin de confirmer ou infirmer, peut-être, la thèse (Teyssier, 1982) selon laquelle les divisions dialectales, au Brésil, sont moins géographiques que socioculturelles et les différences de parlers sont plus fortes, dans un lieu donné, entre un homme cultivé et son voisin analphabète qu'entre deux Brésiliens de même niveau culturel, originaires de deux régions distantes l'une de l'autre.

Tout comme la langue, par nature dynamique, les méthodes d'analyse du phénomène linguistique se modifient avec le temps. Si l'APFB, comme tout autre atlas régional, nous permet seulement d'observer des isoglosses sur un seul territoire, avec l'ALiB, devenu aujourd'hui réalité et doté d'une méthodologie plus avancée, il est possible d'établir une comparaison systématique de tous les échantillons et, peut-être, de confirmer que la continuité proportionnelle d'un phénomène ne s'ajoute pas forcément pari passu à une continuité géographique (Leite \& Callou, 2002; Callou \& Leite, 2010).

Compte tenu des facteurs linguistiques et extralinguistiques déterminants de la dynamique linguistique, on dépasse aussi la phase d'investigation purement descriptive, pour reconnaître que le chercheur ne peut se contenter de décrire la répartition géographique des traits linguistiques. Il doit aussi se soucier d'expliquer - ou peut-être, plus précisément, de fournir des raisons de cette répartition, afin de parvenir à une compréhension du mécanisme sociolinguistique sous-jacent à la répartition géographique des phénomènes linguistiques, de la localisation des isoglosses et 
de la diffusion des innovations. De nos jours, la dialectologie et la sociolinguistique avancent ensemble, pas à pas, dans l'étude de la variation et du changement.

En conclusion, l'Atlas Prévio dos Falares Baianos représente une contribution indéniable au développement de la géolinguistique au Brésil. Il a été le point de départ de l'expansion de la dialectologie dans le pays et l'inspirateur d'autres atlas régionaux réalisés ultérieurement, comme ceux de Sergipe, de Minas Gerais, de Paraíba, pour ne citer que les premiers. Il y a encore beaucoup d'enseignements à tirer de cette œuvre pionnière dont on ne peut nier la pertinence. Les chercheurs y trouvent inspiration et exemple pour leurs recherches qui favoriseront à l'avenir une meilleure connaissance de notre réalité linguistique, du point de vue spatial et social.

\section{RÉFÉRENCES BIBLIOGRAPHIQUES}

Amaral Amadeu, O dialeto caipira, 1920, São Paulo, Casa Editora O livro.

CALlou Dinah \& Leite Yonne, 2010, «La variación del portugués en el Brasil», dans I. Sichra (éd), Atlas sociolinguiístico de pueblos indígenas en América latina, vol. 2, Quito (Équateur), Imprenta Mariscal, p. 553-567.

CArdoso Suzana Alice et al., 2014, Atlas Lingüístico do Brasil, vol. 1 : Introdução, $212 \mathrm{p}$; vol. 2 : Cartas lingüísticas 1, 368 p., Londrina, EDUEL.

CARdoso Suzana Alice, 2012, «Projeto ALiB : o sentido desta caminhada», dans S. A. Cardoso et al. (éds), Vozes do X Workalib. Amostras do português brasileiro, Documentos 3, Salvador, Vento Leste, p. 13-32.

CinTra Luiz Felipe, 1983, Estudos de Dialectologia Portuguesa, Lisboa, Sá da Costa.

CunHa Celso et al., 1972, «Falares Regionais», dans Atlas Cultural do Brasil, MEC/FENAME, Conselho Federal de Cultura.

Ferreira Carlota, 1998, «Atlas Prévio dos Falares Baianos. Alguns aspectos metodológicos», dans V. d. A. Aguilera (éd.), A Geolingüística no Brasil, Londrina, EDUEL, p. 15-30.

Gillieron Jules \& Edmont Edmond, 1902-1910, Atlas linguistique de la France, Paris, Champion.

LACERDa Armando \& HAMmARSTRÖM Göran, 1953, «Transcrição fonética do português normal », Revista do Laboratório de Fonética Experimental, Faculdade de Letras da Universidade de Coimbra, vol. I, p. 119-135.

Leite Yonne \& CAllou Dinah, 2002, Como falam os brasileiros, Rio de Janeiro, Zahar.

Nascentes Antenor, 1953 [1922], O linguajar carioca, Rio de Janeiro, Simões.

NASCEntes Antenor, 1958/1961, Bases para a elaboração do atlas lingüístico do Brasil, vol. 1/vol. 2, Rio de Janeiro, MEC, Casa de Rui Barbosa. 
Pereira Coruja Antônio Alvares, 1852, «Coleção de vocábulos e frases usados na Província de São Pedro do Rio Grande do Sul», dans Revista do Instituto Histório e Geographico do Brasil, vol. XV, Rio, Laemmert, p. 210-240.

PARIs Gaston, 1888, «Les parlers de France», dans J. Gilliéron et A. Rousselot (éds), Revue des patois gallo-romans, t. 2, Paris, Champion, p. 161-175.

Rossi Nelson et al., 1959, «Aspectos do léxico regional da Bahia», IV Colóquio Internacional de Estudos Luso-Brasileiros, Salvador, Universidade Federal da Bahia, tapuscript.

Rossi Nelson, 1963, Atlas Prévio dos Falares Baianos, Rio de Janeiro, Instituto Nacional do Livro, Ministério da Educação e Cultura.

Rossi Nelson, 1965, Atlas Prévio dos Falares Baianos. Introdução. Questionário Comentado. Elenco das respostas transcritas, Rio de Janeiro, Instituto Nacional do Livro, Ministério da Educação e Cultura.

Silva Neto Serafim da, 1952, História da língua portuguesa, Rio de Janeiro, Livros de Portugal.

Teyssier Paul, 1982, História da língua portuguesa, trad. Celso Cunha, São Paulo, Martins Pontes. 